\title{
GxcC connects Rap and Rac signaling during Dictyostelium development
}

\author{
Katarzyna Plak', Douwe Veltman², Fabrizia Fusetti ${ }^{3}$, Jetze Beeksma ${ }^{1}$, Francisco Rivero ${ }^{4}$, Peter JM Van Haastert ${ }^{1}$ \\ and Arjan Kortholt ${ }^{1^{*}}$
}

\begin{abstract}
Background: Rap proteins belong to the Ras family of small G-proteins. Dictyostelium RapA is essential and implicated in processes throughout the life cycle. In early development and chemotaxis competent cells RapA induces pseudopod formation by activating PI3K and it regulates substrate attachment and myosin disassembly via the serine/threonine kinase Phg2. RapA is also important in late development, however so far little is known about the downstream effectors of RapA that play a role in this process.
\end{abstract}

Results: Here we show that cells expressing constitutively active RapA exhibit a high level of Rac activation. With a pull-down screen coupled to mass spectrometry, we identified the Rac specific guanine nucleotide exchange factor, GxcC, as Rap binding partner. GxcC binds directly and specifically to active RapA and binds to a subset of Dictyostelium Rac proteins. Deletion studies revealed that this pathway is involved in regulating Dictyostelium development.

Conclusions: GxcC provides a novel link between Rap and Rac signalling and is one of the Rap effectors regulating the progression of multicellular development.

Keywords: Rap, Rac, GEF, Development, Dictyostelium

\section{Background}

Small G-proteins are molecular switches that cycle between an inactive GDP- and active GTP-bound state. Activation is dependent on helper proteins that facilitate release and exchange of bound nucleotide: GEFs (Guanine nucleotide Exchange Factors), while its deactivation is regulated by GAPs (GTPase activating proteins) that stimulate the naturally low GTPase activity [1]. Only in the active GTP bound form, the switch region of small G-proteins can interact with Ras-associating (RA) or Ras binding domains (RBD) of effector proteins [2,3]. In this way Small G-proteins provide tight control over the cell response to extracellular stimuli by translating the signaling cascades inside the cell [4].

In Dictyostelium discoideum small G-proteins are essential for a wide variety of processes, including regulation of the cytoskeleton, chemotaxis, cell division and multicellular development $[5,6]$. Due to its genetic

\footnotetext{
*Correspondence: A.Kortholt@rug.nl

'Department of Cell Biochemistry, University of Groningen, Nijenborgh 7, Groningen, AG 9747, The Netherlands

Full list of author information is available at the end of the article
}

tractability and high conservation of many important signaling pathways Dictyostelium has proven to be an excellent model for studying small G-protein signaling. During the vegative state, Dictyostelium are single-celled amoeba that feed on bacteria. Upon starvation, cells undergo a tightly regulated developmental process in which they secrete and chemotax toward cAMP, resulting in multicellular fruiting bodies $[7,8]$.

Rap proteins belong to the Ras superfamily of small G-proteins. In mammalian cells Rap is important for cellular adhesion, differentiation and cell proliferation $[9,10]$. Dictyostelium RapA is essential and is implicated in processes throughout the life cycle. In vegetative and chemotaxis competent cells RapA induces pseudopod formation by activating PI3K [11], and it regulates substrate attachment and myosin II disassembly via the serine/threonine kinase Phg2 [12,13]. Strains with a deletion of rapGAP2 or rapGAP3 showed defects in cell patterning and morphogenesis $[14,15]$, indicating that RapA is also important in late development. However so far nothing is known about downstream effectors of 
RapA that play a role in the progression of multicellular development.

Here we show that activation of RapA results in increased level of activated Rac proteins. To gain further insight into the link between these pathways, we have used a proteomic approach. Recombinant RapA was used as bait in pull-down screens and interacting proteins were identified by mass-spectroscopy. One of the binding partners, GxcC provides a potential link between Rap and Rac activation and deletion studies reveal that this pathway is involved in regulating development of Dictyostelium cells.

\section{Results and discussion}

\section{Rap regulates Rac activation}

Rap proteins are involved in the regulation of the actin cytoskeleton. In mammals Rap promotes cell spreading via the RacGEFs Vav2 and Tiam1 [16]. In Dictyostelium expression of constitutive active RapA $\left(\right.$ RapA ${ }^{\mathrm{CA}}$ ) results in an increased actin response and F-actin polymerization is induced at the sites were RapA is activated in response to cAMP [17]. However the pathways and mechanism by which Rap regulates cytoskeleton reorganization are not completely understood. The dynamics of Rac activation in Dictyostelium cells can be monitored using the fluorescent probe CRIB-GFP that consists of the CRIB domain of Dictyostelium PakB, that specifically binds to the active form of Rac $[18,19]$. To test if RapA can indeed activate the Rac pathway we coexpressed RapA ${ }^{\mathrm{CA}}$ and CRIB-GFP and visualised active Rac using total internal reflection microscopy. In random moving wild type (AX3) cells there is a broad patch of active Rac in pseudopods. In unstimulated developed cells, there is usually a single $(1.3 \pm 0.5, \mathrm{n}=$ 20) stable patch that slowly migrates along with the pseudopod (Figure 1A, Additional file 1: Movie 1). Cells expressing $\mathrm{RapA}^{\mathrm{CA}}$ are flattened and unpolarized in appearance [13]. In these cells, active Rac patches are highly motile (Figure 1A, Additional file 2: Movie 2). Patches grow to unusually large size and often break up into multiple $(2.4 \pm 0.9, \mathrm{n}=20)$ patches, which leads to multiple simultaneous protrusions.

\section{GxcC is a specific RapA effector}

To identify Rap effectors we performed a pull-down screen in Dictyostelium lysate with purified RapA loaded with GppNHp as bait. By using mass-spectrometry a putative Rac GEF (GxcC - Guanine eXchange factor for raC, UniProt: Q54P24) was identified as potential binding partner. GxcC has N-terminal ARM repeats, followed by a coiled-coil region, a predicted RhoGEF (DH) domain and a Pleckstrin Homology (PH) domain (Figure 1B). Dictyostelium cells do not have Rho or Cdc42 homologues, but RhoGEF domain containing proteins are thought to regulate the function of the Dictyostelium Rac family [20].

To confirm that RapA can interact with GxcC, we coexpressed GST-fused GxcC and GFP-tagged RapA ${ }^{\mathrm{CA}}$ in Dictyostelium and performed pull-down experiments using GSH beads. Using GST-GxcC as a prey we were able to pull-down GFP-RapA ${ }^{\mathrm{CA}}$, whereas free GFP that is present in the same sample does not bind to $\mathrm{GxcC}$ (Figure 1C).

Mammalian Rap1 binds to the DH-PH tandem of the RacGEFs, TIAM and VAV2 [17,21]. To determine which domain of $\mathrm{GxcC}$ is responsible for RapA binding, we expressed truncated GxcC constructs (Figure 1B) and performed pull down experiments. Figure 1D shows that the DH-PH tandem is not binding to active RapA and that binding between RapA and GxcC is mediated by the ARM domain containing $\mathrm{N}$-terminal fragment. This interaction is not disrupted by the actin inhibitor LantrunculinA, indicating that RapA binding to $\mathrm{GxcC}$ does not need an intact cytoskeleton (Additional file 3: Figure S1A). To determine the specificity of the RapA/GxcC interaction we tested the ability of RasC and RasG to bind to $\mathrm{GxcC}$. GxcC does not bind to active RasG and only very weakly to active RasC (Additional file 3: Figure S1B).

Rap activation in vivo can be monitored with the previously published GFP-RalGDS reporter, which specifically binds to Rap-GTP [22]. Upon uniform stimulation with cAMP, GFP-RalGDS transiently translocates uniformly to the cell boundary, maximum peaking after 3$6 \mathrm{sec}$ after stimulation, followed by a return to the cytoplasm around $20 \mathrm{sec}$ (Figure 2A + B) [13]. N-terminal GxcC shows a highly similar response; it is recruited from the cytoplasm to the cell boundary upon cAMP stimulation, with a maximum drop in the cytoplasm after $5 \mathrm{sec}$ after stimulation and return to basal after 20 sec (Figure $2 \mathrm{~A}+\mathrm{B}$ ). Upon application of a chemoatractant gradient to wild-type cells, both GFP-RalGDS and $\mathrm{N}$-terminal GxcC localize at the side of the cell facing the gradient (Figure 2A). On the contrary, full length GxcC is uniformly distributed in the cytosol and doesn't alter localization upon stimulation with cAMP (Figure $2 \mathrm{~A}+\mathrm{B}$ ), suggesting $\mathrm{GxcC}$ is in an auto-inhibited state and may need additional specific inputs for translocation to the membrane and/or RapA is locally activating GxcC rather than regulating its localization. These data show that the localization and kinetics of RapA activation coincides with the localization of $\mathrm{N}$-terminal $\mathrm{GxcC}$, indicating an interaction in vivo.

To address if the interaction between GxcC and RapA is direct and nucleotide dependent, the ARM domain of GxcC (Figure 1E) and RapA were expressed and purified from E. coli and subsequently used in a Guanine nucleotide dissociation inhibition (GDI) assay. In this 

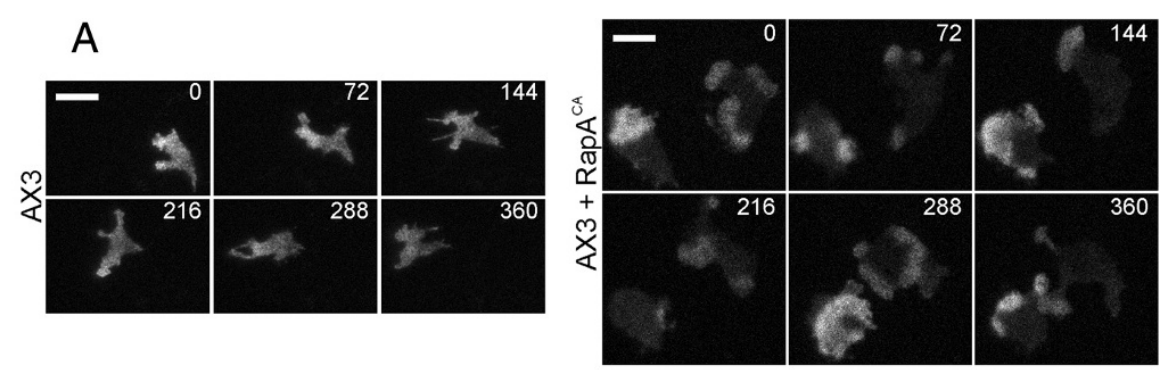

B
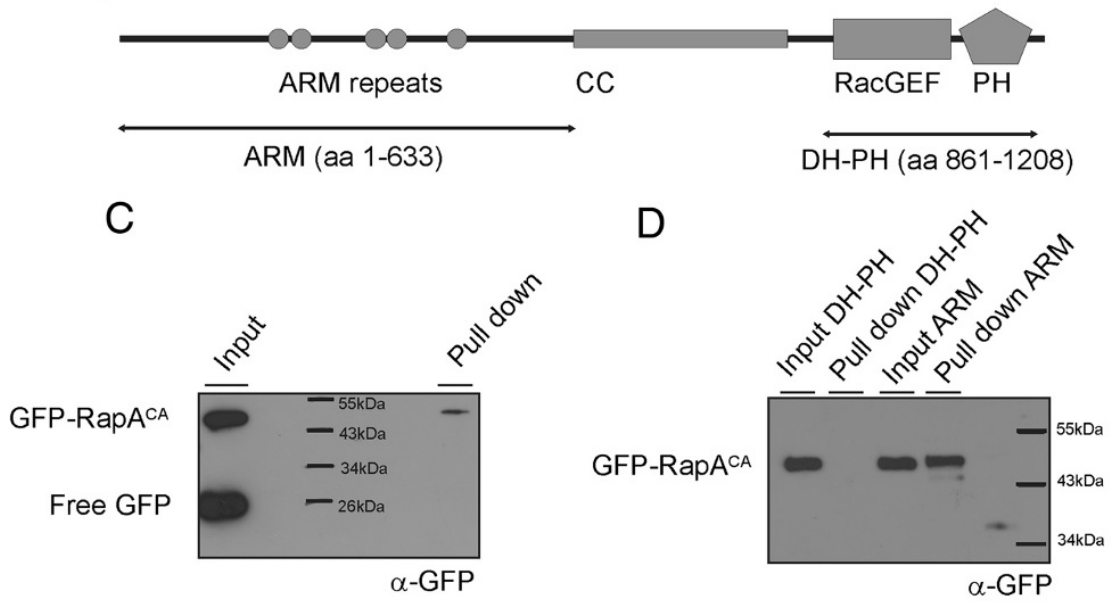

E

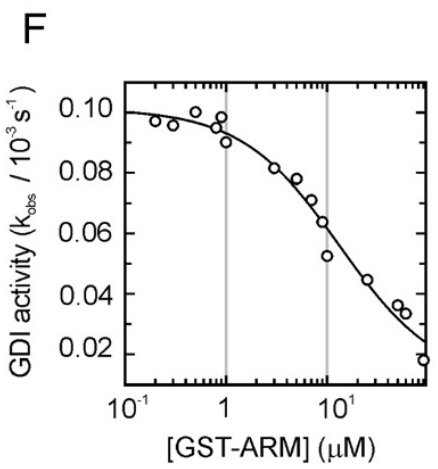

Figure $1 \mathrm{GxcC}$ is a specific RapA interaction partner. (A) Dynamics of Rac activation visualized with CRIB-GFP. Cells were developed in shaken suspension for 3 hours and pulsed with $50 \mathrm{nM}$ cAMP for another 2 hours. Developed cells were transferred to a glass bottom dish and overlayed with PB + 0.4\% agarose. Images were collected using TIRF microscopy (original experiments are shown in Additional file 1: Movie 1 and Additional file 2: Movie 2). Scale bar is $10 \mu \mathrm{M}$. (B) Domain composition of GxCC. ARM - Armadillo repeats, CC - Coiled Coil region, RacGEF Guanine Nucleotide Exchange factor for Rac, PH - Pleckstrin Homology domain. Arrows indicate the boundaries of truncated constructs. (C) Pull down in Dictyostelium lysate with GST-GXCC as bait and GFP-RapA ${ }^{\text {CA }}$ as prey. (D) Pull down with GST-ARM or GST-DH-PH as bait and GFP-RapA ${ }^{\text {CA }}$ as prey. The amount of prey was detected by western blotting using antibody specific for GFP (E) SDS PAGE analysis of recombinant GST-ARM ( $95 \mathrm{kDa}$ ) purified from $E$ coli. (F) GDI assay in which the dissociation rate of $\mathrm{mGppNHP}$ from RapA was measured in the presence of varying concentrations of GST-ARM. The addition of increasing concentrations of the effector results in a concentration-dependent decrease of the observed rate constant $\left(k_{\text {obs }}\right)$.

experiment RapA loaded with mGppNHp, a hydrolysisresistant fluorescent GTP analogue, is incubated with an excess of unlabelled nucleotide. The resulting nucleotide exchange can be monitored as decay in fluorescence, from which the rate constant $\mathrm{k}_{\mathrm{obs}}$ is calculated. The interaction of an effector with the GTP-bound G-protein stabilizes the interaction between the G-protein and the nucleotide and this stabilization results in decreased dissociation of the nucleotide from the G-protein/ nucleotide/ effector complex [23]. The addition of increasing concentrations of the purified GxcC ARM domain resulted in a concentration dependent decrease of $\mathrm{k}_{\mathrm{obs}}$. Using this dependency we determined an affinity $\left(\mathrm{K}_{\mathrm{d}}\right)$ of 13.3 $\mu \mathrm{M}$ (Figure 1F) for the interaction of RapA and 


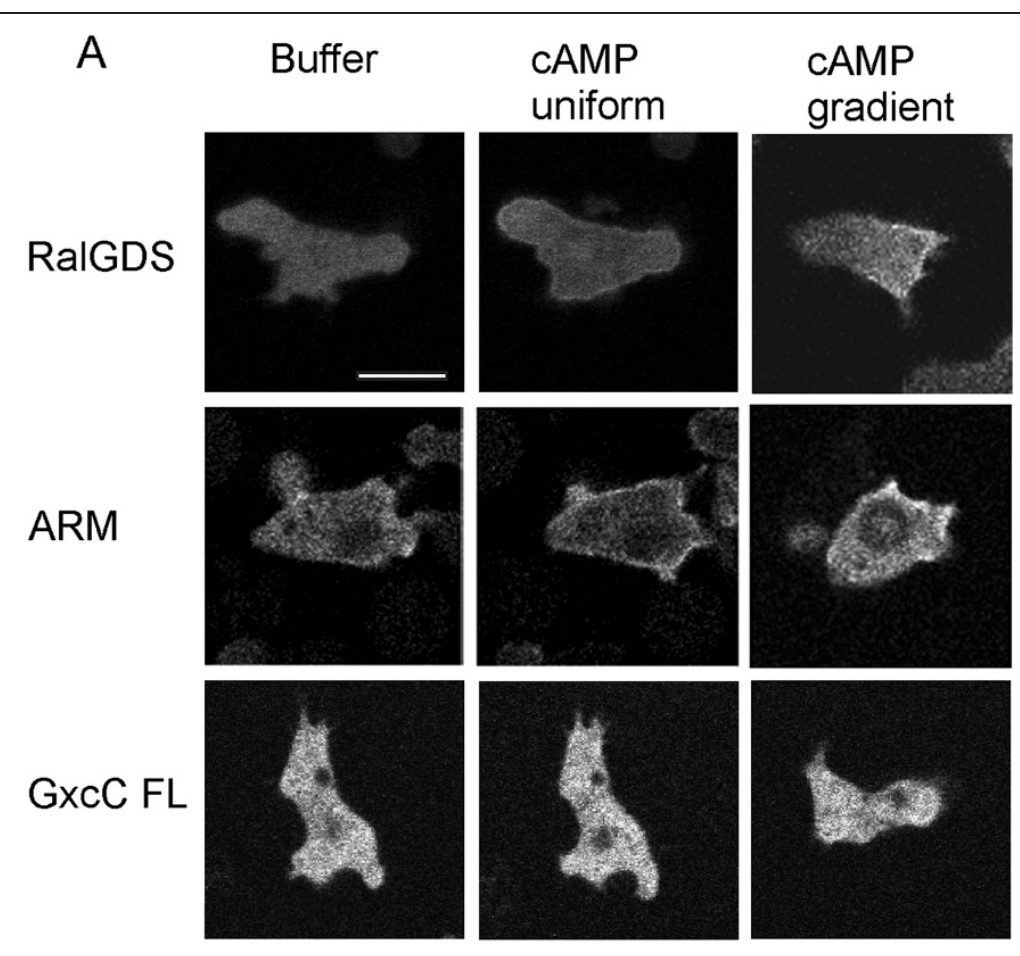

B

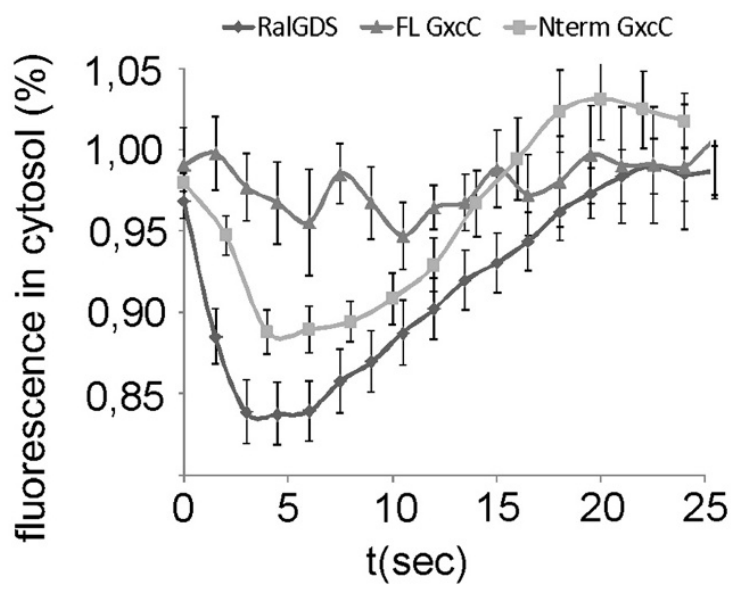

Figure 2 Localization and translocation dynamics of GxcC ARM coincides with Rap activation. (A) Confocal images of starved cells expressing GFP-RalGDS, GFP-ARM or GFP-GxcC in buffer, 4 sec after CAMP stimulation or in a gradient of cAMP (B) Time-course of RalGDS, GFPARM or GFP-FL GXCC translocation from cytoplasm to the membrane upon stimulation with $1 \mu \mathrm{M}$ CAMP. Shown are the mean and SEM of at least 9 cells.

GxcC. Although this affinity seems to be rather low, it is similar to the ones described for the interaction between RapA and Phg2 [12], RapA and PI3K [11] and human Rap with several effectors [24]. Measurements with RapA bound to mGDP or mGppNHp loaded RasC and RasG, didn't show any GDI effect in the presence of $\mathrm{GxcC}$, indicating the interaction is nucleotide dependent and specific.

All together our results show that active RapA specifically binds to the ARM repeat region of $\mathrm{GxcC}$ in vitro and vivo.

\section{GxcC binds to Rac proteins}

The Dictyostelium genome encodes for 18 Rac proteins, whereas Rho and CDC42 are absent [20]. To determine the downstream targets of $\mathrm{GxcC}$, binding to Dictyostelium Rac proteins was determined. The Rac proteins were expressed and purified from E. coli, bound to GSH beads and subsequently incubated with lystate of vegetative GFPGxcC Dictyostelium cells in the presence of EDTA. Imunnoblotting was used to detect bait (GST-Rac) and prey (GFP-GxcC) protein. GxcC showed the highest affinity to RacG, RacH, RacE, RacI and RacL whereas hardly binding 
to Rac1A, RacF2 and RacC could be detected (Figure 3), indicating specificity of $\mathrm{GxcC}$ binding in the pull-down. To get complete insight in the downstream targets of $\mathrm{GxcC}$ we tried to perform in vitro nucleotide exchange assays, but unfortunately we were not able to isolate a stable recombinant GxcC DH-PH fragment. We were able to isolate small quantities of full length GxcC from Dictyostelium cells, but this protein didn't show exchange activity on any of the tested Rac proteins. This lack of activity could be due to the quality of the isolated protein, or consistent with the translocation data may suggest that full length $\mathrm{GxcC}$ is in an auto-inhibited state (unpublished results).

Only a few of the Rac proteins have been characterized in detail. RacG which shows the highest binding is involved in cAMP chemotaxis, filopodia formation and phagocytosis [25]. RacH which also shows high binding to GxcC has a role in vesicular trafficking [26], RacE is known to play a role in orchestrating cell division [27], and RacL is a yet uncharacterized Rac protein that is expressed at late stages of Dictyostelium development [28].

Together the data shows that $\mathrm{GxcC}$ can bind specific Rac proteins. The presence of the predicted RhoGEF $\mathrm{DH}-\mathrm{PH}$ domain suggest that $\mathrm{GxcC}$ most likely is able to activate these interacting proteins, it is however also unlikely that it is the only exchange factor for these Rac's.

\section{GxcC plays a role during development}

To characterize the function of $\mathrm{GxcC}$ in vivo we generated cells lacking $g x c C$ by homologous recombination.
Three independent knock out clones have been isolated and the integration in the proper loci was confirmed by PCR (Additional file 3: Figure S2). A rescue cell line was prepared by overexpressing a full length $\mathrm{GxcC}$ construct in the knock out background. The role of $\mathrm{GxcC}$ in chemotaxis was tested using a micropipette assay. In a gradient of cAMP, wild type cells were very polarized and robustly moved towards the pipette with a chemotaxis index of $0.79 \pm 0.09$ and speed of $9.57 \pm 2.97 \mu \mathrm{m} /$ min. Cells lacking $g x c C$ migrate with similar efficiency (chemotaxis index of $0.81 \pm 0.07$ ), and move slightly faster (speed of $15.2 \pm 3.34 \mu \mathrm{m} / \mathrm{min}$ ), as wild-type cells. The knock out cells did not show defects in cytokinesis, also the cell attachment and cell morphology of $g x c C$-null and $\mathrm{GxcC}$ rescue cells is similar to that of wild-type, suggesting that $\mathrm{GxcC}$ does not play an important regulatory role in these processes. Consistently, gxcC-null cells expressing RapA ${ }^{C A}$ still possessed the characteristic Rap $^{\mathrm{OE}}$ phenotype; cells were flat, highly adhesive extended many substrate attached pseudopodia with highly mobile Rac patches (unpublished results).

Because Rap activation is required for proper cell sorting and fruiting body formation $[14,15]$, we examined the phenotype of $g x c C$-null during Dictyostelium development. In wild-type (AX3), aggregation centers were formed after 6 hours, slugs were formed after 15 hours, Mexican hats were visible after 18 hours and after 24 hours cells culminates into fruiting bodies (Figure 4). Cells lacking $g x c C$ formed territories and

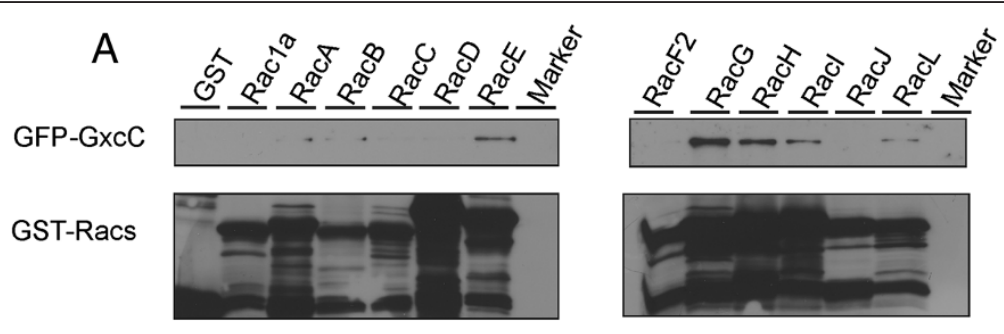

B

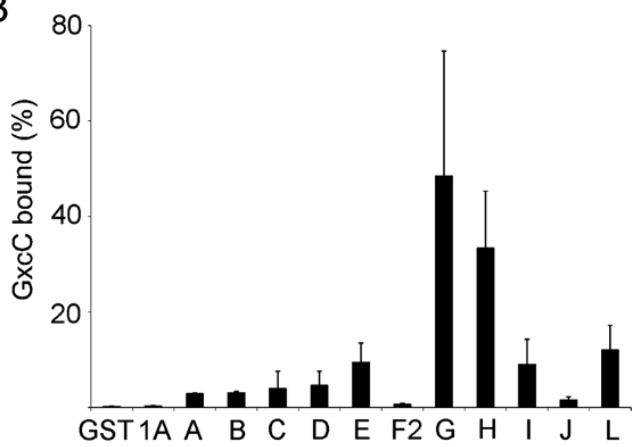

Figure $3 \mathrm{GxcC}$ binds to a subset of Dictyostelium Rac proteins. (A) GST and various purified GST-Rac proteins were incubated with GFP-GxcC cell lysate. Beads were precipitated and the amount of GFP-GXCC and bait was detected by western blotting using antibody specific for GFP or GST, respectively (B) The amount of detected proteins was quantified using ImageJ and normalized to the amount of GxcC protein used in the assay. Shown is the percentage of bound GxcC as mean and SEM of three independent experiments. 


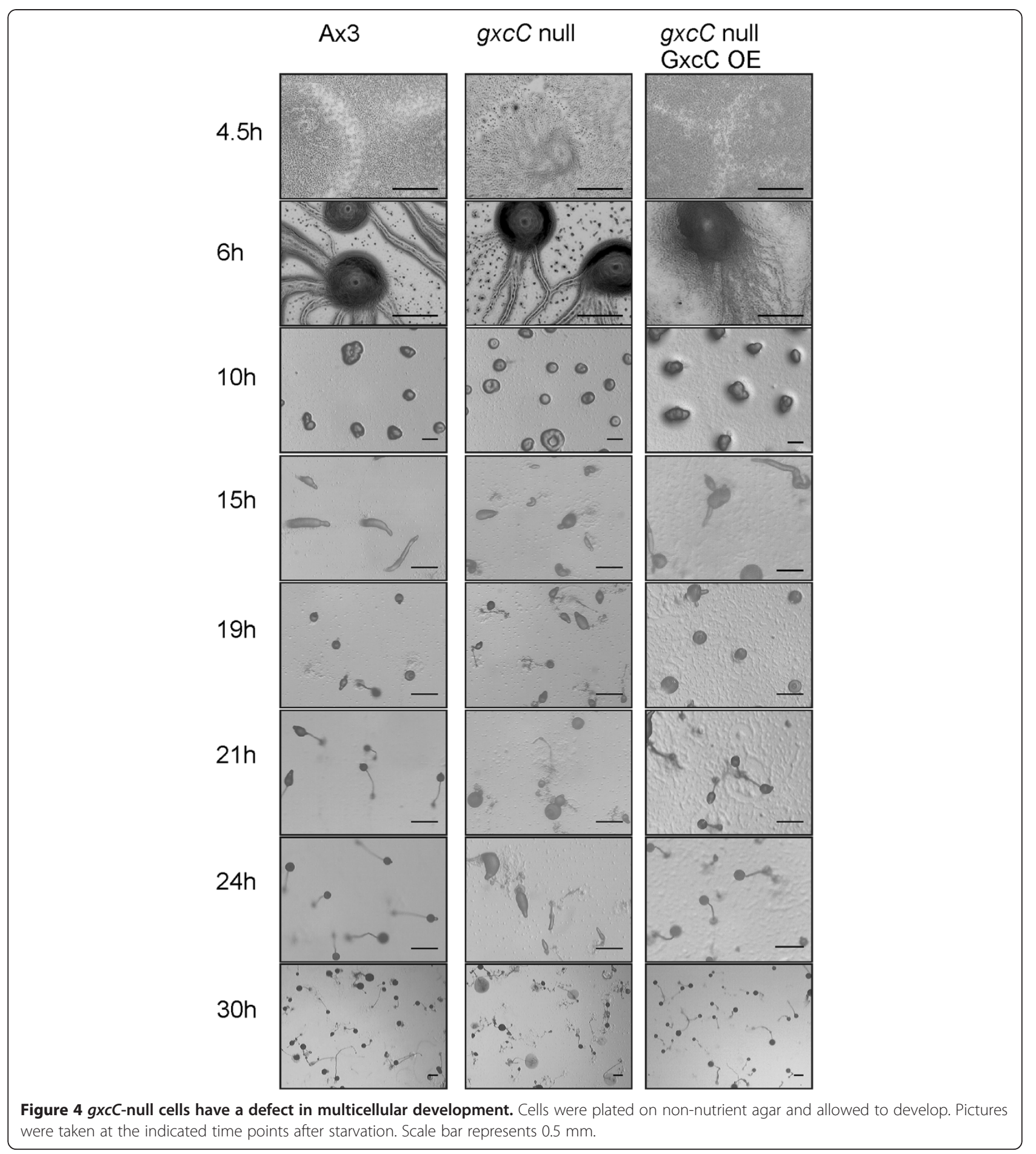

finished streaming phase at the same time as wildtype parent. However, they were severely delayed in forming slugs and Mexican hat structures, and eventually formed fruiting bodies after 30 hours of starvation. This developmental defect could be completely reverted by re-expressing $\mathrm{N}$-terminal GFP tagged GxcC (Figure 4).
Together the data shows that GxcC plays a role in the regulation of Dictyostelium development.

\section{Conclusions}

With a mass spectrometry based screen we here identified the Rac guanine nucleotide exchange factor, $\mathrm{GxcC}$ as Rap binding partner. Our biochemical data shows that 
the interaction between GxcC and RapA is both specific and direct, suggesting a potential link between Rap and Rac signalling. In mammalian cells Rap promotes cell spreading by localization of the RacGEFs Vav2 and Tiam [16]. Dictyostelium Rap is a key regulator of adhesion, chemotaxis and development $[11,13,15]$. GxcC only regulates Dictyostelium late development and is not required for adhesion or chemotaxis. Starved cells expressing Rap ${ }^{\mathrm{CA}}$ have increased Rac activation, suggesting there has to be additional modes of Rap regulation of the Rac pathway. A recent paper by Jeon et al., suggests that Dictyostelium RacGEF1 might be one of these connection between Rap and Rac signalling [17]. To get more insight in these pathways it will be important to identify further regulators and downstream effectors of small G-protein signaling; our mass-pull-down screen could be important in this enterprise.

\section{Methods}

\section{Cell culture and preparation}

AX3 Dictyostelium cells were used as wild-type strain. Cells were grown in HL5-C media (Formedium) either on nunclon-coated petri dishes or in shaking flasks. The indicated GxcC overexpressing and knock out constructs were transformed to Dictyostelium cells. For selection, the media was supplemented with the appropriate antibiotics; $10 \mu \mathrm{g} / \mathrm{ml}$ Blasticidine $\mathrm{S}$ for $\mathrm{KO}$ cell lines, and $10 \mu \mathrm{g} / \mathrm{ml}$ Genticin or $50 \mu \mathrm{g} / \mathrm{ml}$ Hygromycine B for overexpression cell lines. To induce starvation cells were washed with $\mathrm{PB}$ buffer (10 mM KH $\mathrm{HO}_{4} / \mathrm{Na}_{2} \mathrm{HPO}_{4}$, $\mathrm{pH}$ 6.5) and plated out on layers of non-nutrient agar (1,5\% agar in $\mathrm{PB})$.

\section{Construction of plasmids}

The $g x c C$ gene was amplified from cDNA by PCR using the primers sequences: 5'-GGGATCCATGCCAATTC AATTTGATGC-3' and $5^{\prime}$-GCTAGCTTATTTTTTTGA AGTTAAAACTTTTG-3'. An N-terminal construct of GxcC (base pairs 1-1911) was amplified using the forward primer 5'-GGATCCATGCCAATTCAATTTG ATGC-3' $3^{\prime}$ and reverse primer $5^{\prime}$-GCTAGCTTCAGC TTCATCTTCATCATCTTC-3'). A C-terminal GxcC (basepairs 2578-3648) construct was obtained with the primers: $5^{\prime}$-GGATCCGATCAACAAGATAAAGCT TCAC3 ${ }^{\prime}$ and 5'GCTAGCTTATTTTTTTGAAGTTA AAACTTTTG-3'). The obtained PCR fragments were digested with BamHI and ligated into the BglII site of the previously characterized Dictyostelium extrachromosomal plasmids pDM314 (N-terminal GST) and pDM317 (N-terminal GFP) [29]. For expression in E. coli the digested $\mathrm{N}$-terminal $\mathrm{GxcC}$ fragment was ligated into the BamHI site of pGEX 4T3 (GE Healthcare, N-terminal GST).

The $g x c C$ gene disruption construct was created by amplifying the GxcC fragment from position 1660 to
3648 (5'-GGATCCAGAAATCGTAAGGTTATGAATG-3', 5'-GCTAGCTTATTTTTTTGAAGTTAAAACTTTTG-3'). This fragment was ligated in a pBluescript (Stratagene) vector and a BSR cassette was inserted in the Eco72I site of the resulting vector. The $\mathrm{KO}$ fragment was linearized by restriction digestion and isolated.

\section{RapA pull down screen}

GST-RapA fusion protein was purified as described before [12]. The purified protein was preloaded with nonhydrolyzable GTP analogue GppNHp and 3-5 $\mathrm{mg}$ of protein was prebound to a GSH-column. Lysate of Dictyostelium discoideum vegetative cells was circulated over the column overnight to allow binding of possible interaction partners to the activated GST-RapA protein. Unbound proteins were washed away from the column and RapA and its interaction partners were subsequently eluted with glutathione elution buffer.

\section{Protein identification by mass spectrometry}

Protein samples were concentrated and separated by 1D-SDS-PAGE. After Coomassie staining each lane was cut into 24 and subjected to in-gel digestion with trypsin (Trypsin Gold, Promega), prior reduction with $10 \mathrm{mM}$ DTT and alkylation with $55 \mathrm{mM}$ iodoacetamide. Peptide mixtures were trapped on C18 reversed phase EASYColumn and separated on a $100 \mathrm{~mm} \mathrm{C18}$ reversed phase column $(75 \mu \mathrm{m} \times 100 \mathrm{~mm}, 3-\mu \mathrm{m}$ particle size, Thermo Scientific) using a linear gradient from $0 \%$ to $35 \%$ B ( $\mathrm{A}=0.1 \%$ formic acid; $\mathrm{B}=100 \%(\mathrm{v} / \mathrm{v})$ acetonitrile, $0.1 \%$ formic acid) over $70 \mathrm{~min}$ at a constant flow rate of $300 \mathrm{~nL} / \mathrm{min}$. Nanoflow LC-MS/MS was performed on an EASYII LC system (Thermo Scientific) coupled to an LTQ-Orbitrap XL mass spectrometer (Thermo Scientific) operating in positive mode. MS scans were acquired in the Orbitrap in the range from 350 to $1800 \mathrm{~m} / \mathrm{z}$, with a resolution of 60,000 (FWHM). The 7 most intense ions per scan were submitted to MS/MS fragmentation (35\% Normalized Collision Energy ${ }^{\mathrm{TM}}$ ) and detected in the linear ion trap. Peak lists were obtained from raw data files using the Proteome Discoverer v 1.3 software. Mascot (version 2.1, MatrixScience) was used for searching against a sequence database obtained by combining the E. coli with the Dictyostelium proteome sequences. The peptide tolerance was set to $40 \mathrm{ppm}$ and the fragment ion tolerance to $2.0 \mathrm{Da}$, using semi-trypsin as protease specificity and allowing for up to 2 missed cleavages. Oxidation of methionine residues, deamidation of asparagine and glutamine, and carboamidomethylation of cysteines were specified as variable modifications. Peptide and protein identifications were further validated with the program Scaffold (Version Scaffold_3.2, Proteome Software Inc., Portland, OR). Protein identifications based on at least 2 unique peptides identified by MS/MS, 
each with a confidence of identification probability higher than $95 \%$, were accepted.

\section{Protein purification and GDI assays}

NtermGxcC (aa 1-633) was expressed from a pGEX4T3 plasmid containing a N-terminal GST side. Recombinant protein was purified by GSH affinity and size exclusion chromatography. The purified protein was used in a GDI assay as previously described [23].

\section{GxcC pull down experiments}

Dictyostelium cells expressing GST tagged bait proteins and GFP tagged prey proteins were collected and lysed in $2 \mathrm{ml}$ of $\mathrm{LB}$ buffer $\left(10 \mathrm{mM} \mathrm{Na}_{2} \mathrm{HPO}_{4} \mathrm{pH} 7.2,1 \%\right.$ Triton $\mathrm{X}-100,10 \%$ glycerol, $150 \mathrm{mM} \mathrm{NaCl}, 10 \mathrm{mM} \mathrm{MgCl}_{2}, 1$ $\mathrm{mM}$ EDTA, $1 \mathrm{mM} \mathrm{Na} \mathrm{VO}_{4}, 5 \mathrm{mM} \mathrm{NaF}$ and protease inhibitor cocktail (Roche). Precleared cell lysate was mixed with GSH beads (GE Healthcare) and incubated with rotation overnight at $4^{\circ} \mathrm{C}$. Unbound proteins were washed away with PBS and prey proteins were detected by Western Blot with $\alpha$-GFP primary antibody (SC9996).

\section{Microscopy}

Confocal images were taken with a Zeiss LSM 510 META-NLO confocal laser scanning microscope with a Zeiss plan-apochromatic $\times 63$ numerical aperture 1.4 objective. Femtotip micropipette filled with $0.1 \mathrm{mM}$ cAMP was introduced to the field of cells to induce chemotaxis towards increasing cAMP gradient. The response to uniform stimulation with cAMP was tested by introducing a broken micropipette filled with $1 \mu \mathrm{M}$ cAMP and quantified using ImageJ software [30]. TIRF microscopy was performed on a Nikon Eclipse TE2000-U that was fitted with a custom TIRF condenser and a Nikon 1.45 NA 100x Plan Apo TIRF objective and an Evolve 512 EMCCD camera.

\section{Rac binding assays}

GST-Rac proteins were expressed and coupled to GSH beads as previously described [20]. Pre-cleared lysate of vegetative Dictyostelium cells overexpressing GFP-GxcC was prepared as described above and incubated with the GSH beads coupled GST-Rac proteins. Binding was allowed at $4^{\circ} \mathrm{C}$ for 1 hour, unbound proteins were washed away, and bait and prey proteins were detected by Western Blot with $\alpha$-GFP antibody (SC9996) and $\alpha$ GST antibody (GE-Healthcare). Image J software was used to quantify binding specificity [30].

\section{Additional files}

Additional file 1: Movie 1. Visualisation of active Rac in Wild Type cells. AX3 cells expressing the active Rac marker CRIB-GFP (vector pDM841) were induced to develop by starving them for 4 hours and pulsing them for 2 hours with $100 \mu \mathrm{M}$ cAMP pulses every 6 minutes. Cells were transferred to a glass-bottom dish and overlayed with a thin layer of $0.4 \%$ agarose in development buffer. Fluorescence images (green) were collected using TIRF illumination at 1 frame per 2 seconds. The brightfield image is shown in dark blue. The field of view is $83 \times 83$ micrometers.

Additional file 2: Movie 2. Visualisation of active Rac in RapG12V overexpressing cells. AX3 cells expressing the active Rac marker CRIB-GFP (vector pDM841) and constitutive active Rap (G12V mutation) under a folate-responsive promoter were induced for 24 hours with $1 \mathrm{mM}$ folate. Cells were induced to develop by starving them for 4 hours and pulsing them for 2 hours with $100 \mu \mathrm{M}$ CAMP pulses every 6 minutes. Cells were transferred to a glass-bottom dish and overlayed with a thin layer of $0.4 \%$ agarose in development buffer.

Additional file 3: Figure S1. GxcC is a specific RapA interaction partner. (A) Binding between GxcC and active RapA is independent of filamentous actin. Pull down with GST-RapA ${ }^{\text {CA }}$ as bait and GF-PARM as prey. Dictyostelium cells overexpressing bait and prey proteins were incubated in PB or PB with $5 \mu \mathrm{M}$ Latrunculin A (Sigma) for 10 minutes prior to lysis. The amount of prey was detected by western blotting using antibody specific for GFP. (B) GxcC binds specifically to RapA. Pull down with GST-RapA ${ }^{\text {CA }}$, GST-RasC ${ }^{\text {CA }}$ GST-RasG ${ }^{\text {CA }}$ or GST alone as bait and GF-PARM as prey. Western Blot with GFP antibody was used to detect bound ARM. Figure S2. Construction of gxcC KO cell line. (A) Plasmid map of the gxcC KO construct. BSR cassette was inserted at position 2630. Indicated primers were subsequently used for testing in locus integration. (B) gxcC KO construct was integrates in locus. Three independent clones show 2165bp high band corresponding to disrupted gxcC gene. Control sample of AX3 wt cells DNA shows a band of 1077bp, which indicates undisrupted $g \times c$.

\section{Authors' contributions}

$\mathrm{KP}, \mathrm{PJMvH}$, and AK designed the experiments and wrote the paper. KP performed most biochemical and cellular experiments. JB carried out initial biochemical and localization experiments, DV did the in vivo Rac activation experiments and FF the MS analysis. FR provided constructs and helped with the GST-Rac pull-downs. All authors read and approved the final manuscript.

\section{Authors' information}

The authors declare that they have no competing interest. Supplementary Information is linked to the online version of the paper. Correspondence and request for materials should be addressed to A.Kortholt@rug.nl.

\section{Author details}

'Department of Cell Biochemistry, University of Groningen, Nijenborgh 7, Groningen, AG 9747, The Netherlands. Beatson Institute for Cancer Research, Garscube Estate, Switchback Road, Glasgow G61 1BD, UK. ${ }^{3}$ Department of Biochemistry, Netherlands Proteomics Centre, Groningen Biomolecular Sciences and Biotechnology Institute \& Zernike Institute for Advanced Materials, University of Groningen, Groningen, AG 9747, The Netherlands. ${ }^{4}$ Centre for Cardiovascular and Metabolic Research, The Hull York Medical School and Department of Biological Sciences, University of Hull, Hull HU6 7RX, UK.

Received: 20 September 2012 Accepted: 25 January 2013 Published: 30 January 2013

\section{References}

1. Bourne HR, Sanders DA, McCormick F: The GTPase superfamily: conserved structure and molecular mechanism. Nature 1991, 349:117-127.

2. Nassar N, Horn G, Herrmann C, Scherer A, McCormick F, Wittinghofer A: The 2.2 A crystal structure of the Ras-binding domain of the serine/threonine kinase c-Raf1 in complex with Rap1A and a GTP analogue. Nature 1995, 375:554-560.

3. Vetter $\mathbb{R}$, Wittinghofer $\mathrm{A}$ : The guanine nucleotide-binding switch in three dimensions. Science 2001, 294:1299-1304.

4. Mitin N, Rossman KL, Der CJ: Signaling interplay in Ras superfamily function. Curr Biol 2005, 15:R563-R574.

5. Xiong H, Rivero F: Assaying Rho GTPase-dependent processes in Dictyostelium discoideum. Methods Mol Biol 2012, 827:381-392. 
6. Noegel AA, Schleicher M: The actin cytoskeleton of Dictyostelium: a story told by mutants. J Cell Sci 2000, 113(Pt 5):759-766.

7. Kessin RH: Evolutionary biology. Cooperation can be dangerous. Nature 2000, 408:917-919.

8. Pan P, Hall EM, Bonner JT: Folic acid as second chemotactic substance in the cellular slime moulds. Nat New Biol 1972, 237:181-182.

9. Bos JL: Linking Rap to cell adhesion. Curr Opin Cell Biol 2005, 17:123-128.

10. Bos JL, de Rooij J, Reedquist KA: Rap1 signalling: adhering to new models. Nat Rev Mol Cell Biol 2001, 2:369-377.

11. Kortholt A, Bolourani P, Rehmann H, Keizer-Gunnink I, Weeks G, Wittinghofer A, Van Haastert PJ: A Rap/phosphatidylinositol 3-kinase pathway controls pseudopod formation [corrected]. Mol Biol Cell 2010, 21:936-945.

12. Kortholt A, Rehmann H, Kae H, Bosgraaf L, Keizer-Gunnink I, Weeks G, Wittinghofer A, Van Haastert PJ: Characterization of the GbpD-activated Rap1 pathway regulating adhesion and cell polarity in Dictyostelium discoideum. J Biol Chem 2006, 281:23367-23376.

13. Jeon TJ, Lee DJ, Merlot S, Weeks G, Firtel RA: Rap1 controls cell adhesion and cell motility through the regulation of myosin II. J Cell Biol 2007 176:1021-1033.

14. Jeon TJ, Lee S, Weeks G, Firtel RA: Regulation of Dictyostelium morphogenesis by RapGAP3. Dev Biol 2009, 328:210-220.

15. Parkinson K, Bolourani P, Traynor D, Aldren NL, Kay RR, Weeks G, Thompson CR: Regulation of Rap1 activity is required for differential adhesion, celltype patterning and morphogenesis in Dictyostelium. J Cell Sci 2009, 122:335-344.

16. Arthur WT, Quilliam LA, Cooper JA: Rap1 promotes cell spreading by localizing Rac guanine nucleotide exchange factors. I Cell Biol 2004, 167:111-122.

17. Mun H, Jeon TJ: Regulation of actin cytoskeleton by Rap1 binding to RacGEF1. Mol Cells 2012, 54:71-76.

18. de la Roche M, Mahasneh A, Lee SF, Rivero F, Cote GP: Cellular distribution and functions of wild-type and constitutively activated Dictyostelium PakB. Mol Biol Cell 2005, 16:238-247.

19. Veltman DM, King JS, Machesky LM, Insall RH: SCAR knockouts in dictyostelium: WASP assumes SCAR's position and upstream regulators in pseudopods. J Cell Biol 2012, 198:501-508.

20. Mondal S, Neelamegan D, Rivero F, Noegel AA: GxcDD, a putative RacGEF, is involved in dictyostelium development. BMC Cell Biol 2007, 8:23.

21. Raaijmakers JH, Bos JL: Specificity in Ras and Rap signaling. J Biol Chem 2009, 284:10995-10999.

22. Kortholt A, van Haastert PJ: Highlighting the role of Ras and Rap during dictyostelium chemotaxis. Cell Signal 2008, 20:1415-1422.

23. Herrmann C, Horn G, Spaargaren M, Wittinghofer A: Differential interaction of the ras family GTP-binding proteins H-Ras, Rap1A, and R-Ras with the putative effector molecules Raf kinase and Ral-guanine nucleotide exchange factor. J Biol Chem 1996, 271:6794-6800.

24. Wohlgemuth S, Kiel C, Kramer A, Serrano L, Wittinghofer F, Herrmann C: Recognizing and defining true Ras binding domains I: biochemical analysis. J Mol Biol 2005, 348:741-758.

25. Somesh BP, Vlahou G, lijima M, Insall RH, Devreotes P, Rivero F: RacG regulates morphology, phagocytosis, and chemotaxis. Eukaryot Cell 2006 5:1648-1663.

26. Somesh BP, Neffgen C, lijima M, Devreotes P, Rivero F: Dictyostelium RacH regulates endocytic vesicular trafficking and is required for localization of vacuolin. Traffic 2006, 7:1194-1212

27. Larochelle DA, Vithalani KK, De Lozanne A: A novel member of the rho family of small GTP-binding proteins is specifically required for cytokinesis. J Cell Biol 1996, 133:1321-1329.

28. Rot G, Parikh A, Curk T, Kuspa A, Shaulsky G, Zupan B: DictyExpress: a dictyostelium discoideum gene expression database with an explorative data analysis web-based interface. BMC Bioinformatics 2009, 10:265

29. Veltman DM, Akar G, Bosgraaf L, Van Haastert PJ: A new set of small, extrachromosomal expression vectors for dictyostelium discoideum. Plasmid 2009, 61:110-118.

30. Abramoff MD, Magalhaes PJ, Ram SJ: Image processing with ImageJ. Biophotonics International 2004, 11:36-42.

doi:10.1186/1471-2121-14-6

Cite this article as: Plak et al:: GxcC connects Rap and Rac signaling during Dictyostelium development. BMC Cell Biology 2013 14:6.

\section{Submit your next manuscript to BioMed Central and take full advantage of:}

- Convenient online submission

- Thorough peer review

- No space constraints or color figure charges

- Immediate publication on acceptance

- Inclusion in PubMed, CAS, Scopus and Google Scholar

- Research which is freely available for redistribution 